\title{
COVID-19 AS AN OXYGEN-DEPRIVATION DISEASE
}

\author{
Stanislav Yanev* \\ Institute of Neurobiology, Department of Drug Toxicology, Bulgarian Academy of Sciences, \\ Sofia, Bulgaria
}

Since the last year, the epidemic with the new coronavirus SARS-CoV-2 has accumulated a wealth of data. The genetic structure of the virus, the epidemiological characteristics of the spread, the main clinical manifestations, the risks of the healing process, and the approaches for therapeutic intervention, etc. are the main areas into which the information can be parsed. The experimental and clinical data confirm the view that the new coronavirus causes pathogenic changes, which can be summarized as hypoxic syndrome. In this Dance Round, I shall succinctly justify this claim. Biomed Rev 2020; 31: 113-116

Keywords: SARS-CoV-2, hemoglobin, iron, hypoxia

\section{BEFORE THE ONSET OF VIRAL ATTACK}

Coronavirus disease-19 (COVID-19) was regarded as an infective-inflammatory viral disease, which mainly affected lungs. SARS-CoV-2 virus attacked cells containing angiotensin-converting enzyme2 (ACE2), particularly surfactantproducing alveolar type II cells. The clinical signs from this attack are dry cough, fever and most importantly: difficulty breathing, lack of ,air“"

A careful analysis of the clinical picture of "oxygen deprivation" in a number of seriously ill people strongly suggests that the functions of hemoglobin and iron are also marred in the pathogenesis of COVID-19. One of the main physiologic roles of iron is to serve as a component of hemoglobin, the carrier of oxygen to body tissues.
Hemoglobin is composed of four subunits, $2-\alpha$ and $2-\beta$, and each subunit has an iron-bound heme. When iron is divalent (reduced), hemoglobin can release carbon dioxide and capture oxygen atoms in alveolar cells. When hemoglobin reaches other parts of the body through blood circulation, it can release oxygen molecule and capture carbon dioxide.

I hope that this short description of oxygen, the elixir of life, and its journey to the diverse cells of the human body, serves as a foundation for understanding the pathogenesis in COVID-19.

\section{THE VIRUS IS ALREADY AT OUR “FRONT" DOOR}

Here's how SARS-CoV-2 comes in action. The transcribed, non-structural SARS-CoV-2 proteins ORF (Open Reading

* Correspondence to: E-mail: YanevSG@gmail.com

Received 10 December 2020, revised 19 December 2020, accepted 19 December 2020. 
Frame) 8, 3a, 10 play critical roles during infection (viral replication) and disease pathogenesis (stimulate NF-kB mediated inflammation and immune responses). ORF proteins interact with 1-beta chain of hemoglobin $(\mathrm{BCH})$, which leads to the malfunction of heme. As a consequence, iron ion is "disassociated" (released) from the hemoglobin protein $(1,2)$. This is bad for several reasons:

- Without the iron ion, hemoglobin can no longer bind to oxygen. The end result is a lot like carbon monoxide poisoning, in which $\mathrm{CO}$ is bound to the hemoglobin, making it unable to carry oxygen. In such cases, ventilators aren't treating the root cause; the patient's lungs aren't "tiring out", they're pumping just fine. The red blood cells just can't carry $\mathrm{O}_{2}$. The body compensates for this lack of $\mathrm{O}_{2}$ carrying capacity by having the kidneys release hormones like erythropoietin, which tell our bone marrow factories to ramp up production of new red blood cells with fully functioning hemoglobin.

- The released 'free iron ion' is highly reactive, and it increases interleukin 6 (IL-6) levels, causing oxidative damage and inflammation. During active infection, increased ferritin level signifies a vital host defense mechanism that attempts to remove viral growth and defends immune cell function.

- Free iron attacks the liver cells, and hampered its efforts to remove the free iron 'safely'. The result of this "tussle" is the release of higher alanine aminotransferase (ALT) amounts.

- An important diagnostic detail is the picture of the lung. Viral inflammation is diffuse in both lobes of the lungs. Normally, pneumonia develops in one lobe.

It is worth mentioning that there are published in vitro docking data demonstrating the possible interaction of SARSCoV-2 spike protein (S1-RBD) with FDA-approved iron oxide nanoparticles (IONPs) as drugs for anemia treatment (3). This information indirectly enabled the process of tracking hemoglobin iron "released" in the coronavirus pathology.

\section{WHAT ARE THE MOST COMMON CLINICAL SIGNS OF COVID-19 OBSERVED BY THE ATTENDING PHYSICIAN?}

In 48 hours or so, COVID-19 causes prolonged and progressive hypoxia (starving the body of oxygen) by decapacitating hemoglobin in patient's red blood cells. Patients desaturate oxygen (losing $\mathrm{O}_{2}$ in their blood), and this is what eventually leads to fatal organ failures, not any form of acute respiratory distress syndrome (ARDS) or pneumonia.
Prognostic significance of hemoglobin level in SARSCoV-2 infection: Patients with lower hemoglobin levels $(\mathrm{Hgb}<12.5 \mathrm{~g} / \mathrm{dL})$ admitted in intensive care units (ICU) had a higher mortality compared with non-anemic ICU patients (4). This fact indicates that monitoring changes in the level of hemoglobin during the therapeutic process would have great prognostic significance for the outcome of therapy (5). It is important to note that patients with SARS-CoV-2 with reduced iron content in physiologically significant active components (such as: serum iron, ferritin, and transferrin total iron-binding capacity) pose increased risk of pathological changes in the cardiovascular, hepatic and renal systems (6). In a clinical trial with two groups of patients suffering from pneumonia, the group of patients showing PSR positive test (that are COVID-19 positive group) have several times higher levels of serum porphyrin (7).

All the damage to the lungs seen in CT scans are from the release of oxidative iron from the hemes. This overwhelms the natural defenses against pulmonary oxidative stress and causes the always-bilateral ground glass opacity in the lungs.

A huge meta-analysis from 6 bibliographic databases included 189 unique studies, with data from 57563 COVID-19 patients, evaluated biomarkers of anemia and iron metabolism (hemoglobin, ferritin, transferrin, soluble transferrin receptor, hepcidin, haptoglobin, unsaturated iron-binding capacity, erythropoietin, free erythrocyte protoporphyrine, and erythrocyte indices). It showed a correlation of lower hemoglobin and increased ferritin levels with increasing proportion of mortality in second group (8).

An interesting question would be whether abnormalities of $\mathrm{BCH}$, quantitative in thalassemias, and qualitative in Sickle-Cell-Disease (SCD), influence the outcome of virus attack? Only a few confirmative data is available in this regard (9). From 18350 Iranian beta-thalassemic patients only fifteen confirmed cases reported mild to moderate disease of COVID-19 (10).

\section{WHAT THERAPEUTIC APPROACHES DOES THE CURRENT PARADIGM OFFER?}

- Quick blood transfusion with fresh red cells and hemoglobin. As therapies used for anyone with abnormal hemoglobin or malfunctioning red blood cells.

- Use recombinant erythropoietin (rhEPO) for combating anaemia.

Chloroquine (CHQ) is suspected to bind to DNA and interfere with viral attack on hemoglobin and prevents viral 
ORF proteins from attacking heme and forming porphyrin (11). Hydroxychloroquine (an advanced descendant of regular old chloroquine) lowers the $\mathrm{pH}$ which can interfere with the replication of the virus (see Yanev, Zhelyaskova, Chaldakov's Dance Round in this volume of Biomedical Reviews). Ideally, some form of treatment is needed to inhibit viral growth and replication. In this context, $\mathbf{C H Q}+\mathbf{Z P A K}$ (azithromycin 3 day pak) + ZINC or other retroviral therapies are being studied. Lower the virus, lesser hemoglobin loses its iron, and this in turn, lowers the severity and damage (12).

COVID-19 is a part of the hyperferritinemic syndrome spectrum, like secondary haemophagocytic lymphohistiocytosis, catastrophic antiphospholipid syndrome, adult onset Still's disease, and septic shock. To explore this, two clinical trials are under way to test the efficacy and safety of deferoxamine in comparison with standard treatment or to tocilizumab (NCT04333550, NCT04361032) $(13,14)$. The final results of these studies are awaited.

Iron chelators: By binding the excess iron, several effects were achieved. Iron chelators help to obtain effectively functioning hemoglobin and improve tissue oxygenation. At the same time, they reduce the level of free iron required for virus replication; inhibit endothelial inflammation, prevent pulmonary fibrosis and lung insufficiency via reduction of pulmonary iron accumulation (15). Manipulation of the cellular iron level by iron chelators or via increasing iron exporter ferroportin expression, has been shown to have promising effectiveness as adjuvant antiviral therapy of AIDS (16). Deferoxamine is the only drug form for s.c and i.v administration (17). It can also be helpful with its immunomodulatory affect, in adjunction to antiviral drugs used to treat COVID-19 (18).

The hypoxic state of the body in the conditions of viral infection is further complicated by the oxidative hemolysis of erythrocytes induced by SARS-CoV-2 spike (S) protein binding to broadly expressed CD147 receptors on erythrocytes (19; also see Yanev et al's Dance Round in this volume of Biomedical Reviews). That effect, together with altered calcium signaling from SARS-CoV-2 ion channel activities, may contribute to hypercoagulation, thrombosis, and cardiac remodeling, ultimately resulting in heart failure. Melatonin as effective endogenous antioxidant was proved to be useful, with success in prevention and attenuation of hemoglobinopathies, refractory hypoxemia and myocardial injury during critical COVID-19 infections (19).

- Clinicians must be careful with the emergency ventila- tion! The key principle should be to set low pressure with $\mathrm{O}_{2}$.Max. Protect the lungs and don't tear them up! Give immunity the time to do its job!!

- Spend as much time as possible in the fresh air, no matter where, at home near open windows, in the park or on the mountain. And when you are in such an environment, do not wear the masks so that oxygen exchange is facilitated.

- Anyone can find answers or raise questions related to their own practice and professional awareness. As they say, the floor is yours. I hope the discussion will be useful to everyone, particularly to curious minds, scientists and medical practitioners.

\section{REFERENCES}

1. Liu W, Li H. COVID-19: Attacks the 1-Beta Chain of Hemoglobin and Captures the Porphyrin to Inhibit Heme Metabolism. 2020; https://chemrxiv.org/ndownloader/ files/22283226

2. Wen-zhong L, Hua-lan L, Liu W, Li H. COVID-19 Disease: ORF8 and Surface Glycoprotein Inhibit Heme Metabolism by Binding to Porphyrin. 2020; DOI:10.26434/ chemrxiv.11938173.v2

3. Abo-zeid Y, Ismail NSM, McLean GR, Hamdy NM. A molecular docking study repurposes FDA approved iron oxide nanoparticles to treat and control COVID-19 infection. Eur J Pharm Sci 2020; 153: 105465. DOI: 10.1016/j. ejps.2020.105465

4. Algassim AA, Elghazaly AA, Alnahdi AS, MohammedRahim OM, Alanazi AG, Aldhuwayhi NA, et al. Prognostic significance of hemoglobin level and autoimmune hemolytic anemia in SARS-CoV-2 infection.Ann HematolDOI: $10.1007 / \mathrm{s} 00277-020-04256-3$

5. Lippi G, Mattiuzzi C. Hemoglobin value may be decreased in patients with severe coronavirus disease 2019. Hematol Transfus Cell Ther 2020; 42(2): 116-117. DOI: 10.1016/j. htct.2020.03.001

6. Lv Y, Chen L, Liang X, Liu X, Gao M, Wang Q, et al. Association between iron status and the risk of adverse outcomes in COVID-19. Clin Nutr 2020. DOI: 10.1016/j. clnu.2020.11.033

7. San Juan I, Bruzzone C, Bizkarguenaga M, BernardoSeisdedos G, Laín A, Gil-Redondo R, et al. Abnormal concentration of porphyrins in serum from COVID-19 patients. Br J Haematol 2020; 190(5): e265-e267. DOI: 10.1111/bjh. 17060 
8. Taneri PE, Gómez-Ochoa SA, Llanaj E, Raguindin PF, Rojas LZ, Roa-Díaz ZM, et al. Anemia and iron metabolism in COVID-19: a systematic review and meta-analysis. Eur J Epidemiol 2020; 35(8): 763-773. DOI: 10.1007/ s10654-020-00678-5

9. Torti L, Maffei L, Sorrentino F, De Fabritiis P, Miceli $\mathrm{R}$, Abruzzese E. Impact of SARS CoV-2 in Hemoglobinopathies with Immune Disfunction and Epidemiology. A Protective Mechanism from Beta Chain Hemoglobin Defects? Mediterr J Hematol Infect Dis 2020; 12(1): e2020052-e2020052. DOI: 10.4084/MJHID.2020.052

10. Karimi M, Haghpanah S, Azarkeivan A, Zahedi Z, Zarei T, Akhavan Tavakoli M, et al. Prevalence and mortality in $\beta$-thalassaemias due to outbreak of novel coronavirus disease (COVID-19): the nationwide Iranian experience. Br Haematol 2020; 190(3): e137-e140. DOI: 10.1111/ bjh. 16911

11. Wang M, Cao R, Zhang L, Yang X, Liu J, Xu M, et al. Remdesivir and chloroquine effectively inhibit the recently emerged novel coronavirus (2019-nCoV) in vitro. Cell Res 2020; 30(3): 269-271. DOI: 10.1038/s41422020-0282-0

12. Quiros Roldan E, Biasiotto G, Magro P, Zanella I. The possible mechanisms of action of 4-aminoquinolines (chloroquine/hydroxychloroquine) against SARS-CoV-2 infection (COVID-19): A role for iron homeostasis? Pharmacol Res 2020; 158: 104904. DOI: 10.1016/j. phrs.2020.104904
13. Perricone C, Bartoloni E, Bursi R, Cafaro G, Guidelli GM, Shoenfeld Y, et al. COVID-19 as part of the hyperferritinemic syndromes: the role of iron depletion therapy. Immunol Res 2020; 68(4): 213-224. DOI: 10.1007/ s12026-020-09145-5

14. Vlahakos VD, Marathias KP, Arkadopoulos N, Vlahakos DV. Hyperferritinemia in patients with COVID-19: An opportunity for iron chelation? Artif Organs 2020; DOI: 10.1111/aor.13812

15. Dalamaga M, Karampela I, Mantzoros CS. Commentary: Could iron chelators prove to be useful as an adjunct to COVID-19 Treatment Regimens? Metabolism 2020; 108: 154260. DOI: 10.1016/j.metabol.2020.154260

16. Liu W, Zhang S, Nekhai S, Liu S. Depriving Iron Supply to the Virus Represents a Promising Adjuvant Therapeutic Against Viral Survival. Curr Clin Microbiol Rep 2020: 1-7. DOI: 10.1007/s40588-020-00140-w

17. Abbas A, Mostafa A, Yousof E, Ss A. Use of Iron Chelators to Reduce the Severity of COVID-19. 2020; 4: 1042.

18. Abobaker A. Can iron chelation as an adjunct treatment of COVID-19 improve the clinical outcome? Eur J Clin Pharmacol 2020; 76(11): 1619-1620. DOI: 10.1007/ s00228-020-02942-9

19. Loh D. The potential of melatonin in the prevention and attenuation of oxidative hemolysis and myocardial injury from CD147 SARS-CoV-2 spike protein receptor binding. Melatonin Res 2020; 3(3): 380-416. DOI: 10.32794/ $\operatorname{mr} 11250069$ 